\title{
A Comparison of Test Case Prioritization Criteria for Software Product Lines
}

\author{
Ana B. Sánchez, Sergio Segura and Antonio Ruiz-Cortés \\ Department of Computer Languages and Systems, University of Seville, Spain \\ Email: \{anabsanchez,sergiosegura,aruiz\}@us.es
}

\begin{abstract}
Software Product Line (SPL) testing is challenging due to the potentially huge number of derivable products. To alleviate this problem, numerous contributions have been proposed to reduce the number of products to be tested while still having a good coverage. However, not much attention has been paid to the order in which the products are tested. Test case prioritization techniques reorder test cases to meet a certain performance goal. For instance, testers may wish to order their test cases in order to detect faults as soon as possible, which would translate in faster feedback and earlier fault correction. In this paper, we explore the applicability of test case prioritization techniques to SPL testing. We propose five different prioritization criteria based on common metrics of feature models and we compare their effectiveness in increasing the rate of early fault detection, i.e. a measure of how quickly faults are detected. The results show that different orderings of the same SPL suite may lead to significant differences in the rate of early fault detection. They also show that our approach may contribute to accelerate the detection of faults of SPL test suites based on combinatorial testing.
\end{abstract}

\section{INTRODUCTION}

Software Product Line (SPL) engineering is about developing a set of related software products by reusing a common set of features instead of building each product from scratch. Products in an SPL are differentiated by their features, where a feature defines capabilities and behaviour of a software system. SPLs are often represented through feature models. Feature models capture the information of all the possible products of the SPL in terms of features and relationships among them. Figure 1 shows a sample feature model representing an ecommerce SPL. The automated analysis of feature models deals with the computer-aided extraction of information from feature models. These analyses allow studying properties of the SPL such as consistency, variability degree, complexity, etc. In the last two decades, many operations, techniques and tools for the analysis of feature models have been presented [4].

Product line testing is about deriving a set of products and testing each product [21]. An SPL test case can be defined as a product of the product line to be tested, i.e. a set of features. The high number of feature combinations in SPLs may lead to thousands or even millions of different products, e.g. the e-shop model available in the SPLOT repository has 290 features and represents more than 1 billion of products [19]. This makes exhaustive testing of an SPL infeasible, that is, testing every single product is too expensive in general. In this context, there have been many attempts to reduce the space of testing through feature-based test selection [7], [15], [20],
[22]. Test case selection approaches choose a subset of test cases according to some coverage criteria. Most common test selection approaches are those based on combinatorial testing [15], [20], [21], [22]. In these approaches test cases are selected in a way that guarantee that all combinations of $t$ features are tested. Other authors have proposed using search-based and grammar-based techniques to reduce the number of test cases while maintaining a high fault detection capability [2], [10].

Test selection techniques have taken a step forward to make SPL testing affordable. However, the number of test cases derived from selection could still be high and expensive to run. This may be especially costly during regression testing when tests must be repeatedly executed after any relevant change to the SPL. In this context, the order in which products are tested is commonly assumed to be irrelevant. As a result, it could be the case that the most promising test cases (e.g. those detecting more faults) are run in last place forcing the tester to wait for hours or even days before staring the correction of faults. In a worse scenario, testing resources could be exhausted before running the whole test suite remaining faults undetected.

Test case prioritization techniques schedule test cases for execution in an order that attempts to increase their effectiveness at meeting some performance goal [5], [16], [24], [25]. Many goals can be defined, for instance, testers may wish to order their test cases in order to achieve code coverage at the fastest rate possible, exercise components in expected frequency of use or increase the rate of fault detection of test cases. Given a goal, several ordering criteria may be proposed. For instance, in order to increase the rate of fault detection, testers could order test cases according to the number of faults detected by the test cases in previous executions of the suite, or according to the expected error-proneness of the components under test. Test case prioritization techniques have been extensively studied as a complement for test case selection techniques in demanding testing scenarios [9], [23].

In this paper, we present a test case prioritization approach for SPLs. In particular, we explore the applicability of scheduling the execution order of SPL test cases as a way to reduce the effort of testing and to improve their effectiveness. To show the feasibility of our approach, we propose five different prioritization criteria intended to maximize the rate of early fault detection of the SPL suite, i.e. detect faults as fast as possible. Three of these criteria are based on the complexity of the products. Hence, more complex products are assumed to be more error-prone and therefore are given higher priority over less complex products, i.e. they are tested first. Another prioritization criterion is based on the degree of reusability 
of products features. In this case, products including the more reused features are given priority during tests. This enables the early detection of high-risk faults that affect to a high portion of the products. Finally, we propose another criterion based on the so-called dissimilarity among products, i.e. a measure of how different two products are. This criterion is based on the assumption that the more different two products are the higher is the feature coverage and the fault detection rate. The proposed prioritization criteria are based on common metrics of feature models extensively studied in the literature. This allowed us to leverage the knowledge and tools for the analysis of feature models making our approach fully automated. Also, this makes our prioritization criteria complementary to the numerous approaches for feature-based test case selection.

For the evaluation of our approach, we developed a prototype implementation of the five prioritization criteria using the SPLAR tool [18]. We selected a number of realistic and randomly generated feature models and generated both random and pairwise-based test suites. Then, we used our fault generator based on the work of Bagheri et al. [2], [10] to seed the features with faults. Finally, we reordered the suite according to the five criteria and we measured how fast the faults were detected by each ordering. The results show that different orderings of the same SPL suite may lead to significant differences in the rate of fault detection. More importantly, the proposed criteria accelerated the detection of faults of both random and pairwise-based SPL test suites in all cases. These results support the applicability and potential benefits of test case prioritization techniques in the context of SPL. We trust that our work will be the first of a number of contributions studying new prioritization goals and criteria as well as new comparisons and evaluations.

The rest of the paper is structured as follows: Section II presents some background about the analysis of feature model, combinatorial SPL testing and test case prioritization. In Section III we propose five prioritization criteria for SPLs. The evaluation of our approach is described in Section IV. Section $\mathrm{V}$ presents the threats to validity of our work. The related works are presented and discussed in Section VI. Finally, we summarize our conclusions and outline our future work in Section VII.

\section{PRELIMINARIES}

\section{A. Automated analysis of feature models}

SPLs are often graphically represented using feature models. A feature model is a tree structure that captures the information of all the possible products of an SPL in terms of features and relationships among them. Figure 1 shows a simplified feature model representing an e-commerce SPL taken from the SPLOT repository [19]. The model depicts how features are used to specify the commonalities and variabilities of the on-line shopping systems that belong to the SPL.

The analysis of feature models consists on examining their properties. This is performed in terms of analysis operations. Among others, these operations allow finding out whether a feature model is void (i.e. it represents no products) whether it contains errors (e.g. dead features) or what is the number of possible feature combinations in an SPL. Catalogues with up to 30 different analysis operations on feature models have
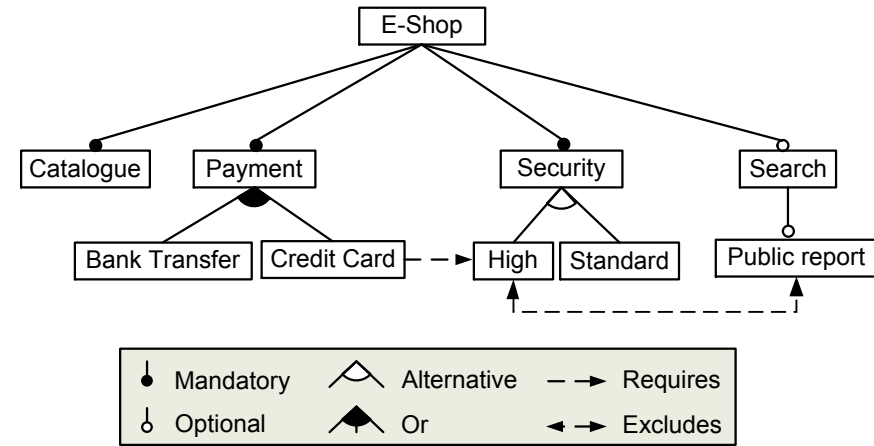

Fig. 1: A sample feature model

been reported in the literature [4]. Some tools supporting the analysis of feature models are AHEAD Tool Suite [1], FaMa Framework [30] and SPLAR [18]. Next, we introduce some of the operations that will be mentioned throughout this paper:

All products: This operation takes a feature model as input and returns all the products represented by the model. For the model in Figure 1, this operation returns the following list of products:

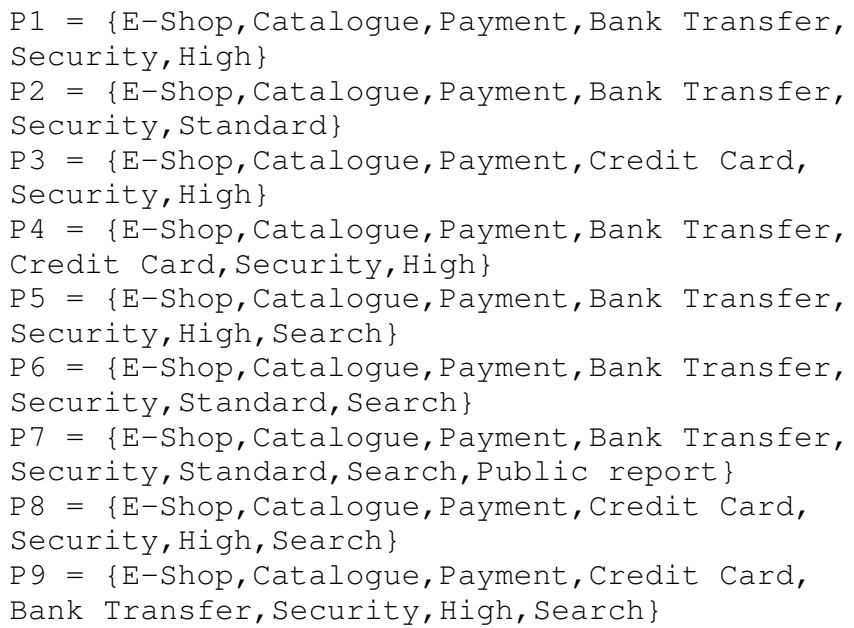

Commonality: This operation takes a feature model and a feature as inputs and returns the commonality of the feature in the SPL represented by the model. Commonality is a metric that indicates the reuse ratio of a feature in an SPL, this is, the percentage of products that include the feature. This operation is calculated as follows:

$$
\operatorname{Comm}(f, f m)=\frac{\text { filter }(f m, f)}{\# \text { products }(\mathrm{fm})}
$$

\#products $(\mathrm{fm})$ returns the number of products of an input feature model, $f m$, and filter $(f m, f)$ returns the number of products in $f m$ that contain the feature $f$. The result of this operation is in the domain [0,1]. As an example, consider the model in Figure 1 and the feature Credit Card. The commonality of this feature is calculated as follows:

$$
\operatorname{Comm}(f, f m)=\frac{\text { filter }(\text { fm,Credit Card })}{\# \text { products }(\text { fm })}=\frac{4}{9}=0.45
$$

The feature Credit Card is therefore included in $45 \%$ of 
the products. A more generic definition of this operation is presented in [4].

Cross-Tree-Constraints Ratio (CTCR): This operation takes a feature model as input and returns the ratio of the number of features (repeated features counted once) in the cross-tree constraints (that are typically inclusion or exclusion constraints) to the total number of features in the model [3], [4], [17]. This metric is usually expressed as a percentage value. This operation is calculated as follows:

$$
C T C R(f m)=\frac{\# \text { constraintsfeatures }(\mathrm{fm})}{\# \text { features }(\mathrm{fm})}
$$

\#constraintsfeatures $(\mathrm{fm})$ is the number of features involved in the cross-tree constraints and \#features $(\mathrm{fm})$ is the total number of features of the model $f m$. The result of this operation is in the domain $[0,1]$. For instance, the CTCR of the model in Figure 1 is $3 / 10=0.3(30 \%)$.

Coefficient of Connectivity-Density (CoC): In graph theory, this metric represents how well the graph elements are connected. Bagheri et al. [3] defined the $\mathrm{CoC}$ of a feature model as the ratio of the number of edges (any connection between two features, including constraints) over the number of features in a feature model. This is calculated as follows:

$$
\operatorname{CoC}(\mathrm{fm})=\frac{\# \text { edges }(\mathrm{fm})}{\# \text { features }(\mathrm{fm})}
$$

\#edges $(\mathrm{fm})$ denotes the number of parent-child connections plus the number of cross-tree constraints of an input model $\mathrm{fm}$ and \#features $(\mathrm{fm})$ is the number of total features in the model $\mathrm{fm}$. For instance, the model in Figure 1 has 11 edges (9 parent-child connections plus 2 constraints) and 10 features, i.e. $\operatorname{CoC}(\mathrm{fm})=11 / 10=1.1$.

Cyclomatic Complexity (CC): The cyclomatic complexity of a feature model can be described as the number of distinct cycles that can be found in the model [3]. Since a feature model is a tree, cycles can only be created by cross-tree constraints. Hence, the cyclomatic complexity of a feature model is equal to the number of cross-tree constraints of the model. In Figure $1, c c(f m)=2$.

Variability Coverage (VC): The variability coverage of a feature model is the number of variation points of the model [10]. A variation point is any feature that provides different variants to create a product. Thus, the variation points of a feature model are the optional features plus all non-leaf features with one or more non-mandatory subfeatures. In Figure $1, v c(f m)=5$ because features E-Shop, Payment, Security, Search and Public report are variation points.

\section{B. Combinatorial SPL testing}

Testing an SPL is a challenging activity compared to testing single systems. Although testing each SPL product individually would be ideal, it is too expensive in practice. In fact, the number of possible products derived from a feature model usually increases exponentially when the number of features grows, leading to thousand or even millions of different products. In this context, there have been many attempts to reduce the space of testing through test selection [7], [15], [20], [22]. The goal of test selection approaches is to reduce the set of feature combinations to a reasonable but representative set of products achieving a high coverage of feature interactions [7]. Most common test selection approaches are those based on combinatorial testing [15], [20], [21], [22]. In these approaches test cases are selected in a way that guarantees that all combinations of $t$ features are tested, this is called t-wise testing [22]. One of the best-known variants of combinatorial testing is the 2-wise (or pairwise) testing approach [15]. This proposal generates all possible combinations of pairs of features based on the observation that most faults originate from a single feature or by the interaction of two features [21]. As an example, Table I depicts the set of products obtained when applying 2-wise selection to the model in Figure 1. The rows of the table represent features and the columns products. An " $\mathrm{X}$ " means that the feature of the row is included in the product of the column, and a gap means that the feature is not included. The number of products to be tested is reduced from 9 to 6 . Oster et al. [20] achieved to reduce the number of products of Electronic Shopping model [19] from $2.26 \cdot 10^{49}$ to 62 products using pairwise coverage.

\begin{tabular}{lcccccc}
\hline Features/Products & P1 & P2 & P3 & P4 & P5 & P6 \\
\hline Bank Trasnsfer & & X & X & X & X & \\
\hline Payment & $\mathrm{X}$ & $\mathrm{X}$ & $\mathrm{X}$ & $\mathrm{X}$ & $\mathrm{X}$ & $\mathrm{X}$ \\
\hline Security & $\mathrm{X}$ & $\mathrm{X}$ & $\mathrm{X}$ & $\mathrm{X}$ & $\mathrm{X}$ & $\mathrm{X}$ \\
\hline High & $\mathrm{X}$ & & & $\mathrm{X}$ & $\mathrm{X}$ & $\mathrm{X}$ \\
\hline Catalogue & $\mathrm{X}$ & $\mathrm{X}$ & $\mathrm{X}$ & $\mathrm{X}$ & $\mathrm{X}$ & $\mathrm{X}$ \\
\hline Public Report & & $\mathrm{X}$ & & & & \\
\hline Credit Card & $\mathrm{X}$ & & & & $\mathrm{X}$ & $\mathrm{X}$ \\
\hline E-Shop & $\mathrm{X}$ & $\mathrm{X}$ & $\mathrm{X}$ & $\mathrm{X}$ & $\mathrm{X}$ & $\mathrm{X}$ \\
\hline Standard & & $\mathrm{X}$ & $\mathrm{X}$ & & & \\
\hline Search & & $\mathrm{X}$ & & $\mathrm{X}$ & $\mathrm{X}$ & $\mathrm{X}$ \\
\hline
\end{tabular}

TABLE I: 2-wise coverage results for SPL in Figure 1

\section{Test case prioritization}

Running all the test cases in an existing test suite can suppose a large amount of effort or even become infeasible due to deadlines and cost constraints. Rothermel et al. [25] reported about an industrial application of 20,000 lines of code whose test suite required seven weeks to be run. For these reasons, various techniques for reducing the cost of testing have been proposed including test case prioritization techniques. Test case prioritization techniques [5], [16], [24], [25] schedule test cases for execution in an order that attempts to increase their effectiveness at meeting some performance goal [25]. There are many possible goals of prioritization [25]. For example, testers may wish to order their test cases in order to reduce the cost of testing (e.g. measuring the testing execution time) or increase the rate of critical fault detection of test cases. Furthermore, given a prioritization goal, various prioritization criteria may be applied to a test suite with the aim of meeting that goal. For instance, in an attempt to increase the rate of fault detection, we could prioritize test cases in terms of the complexity of the system giving priority to the test cases that exercise the most complex components, e.g. those with the higher cyclomatic complexity. Alternatively, we could order test cases according to their coverage running first those test cases that exercise a larger portion of the code. 


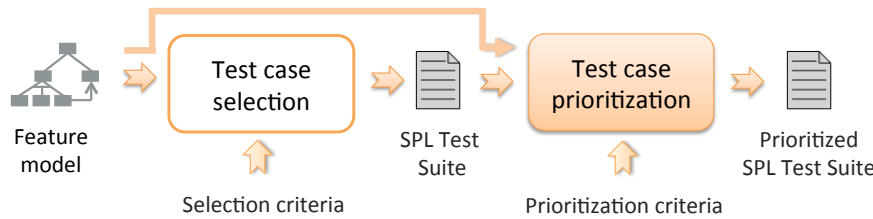

Fig. 2: Overview of the SPL testing process

\section{TEST CASE PRIORITIZATION CRITERIA FOR SOFTWARE PRODUCT LINES}

In this section, we propose the application of test case prioritization techniques in the context of SPL. As a part of the proposal we define and compare five test case prioritization criteria to maximize the rate of early fault detection of a test suite. This goal aims to achieve a sequence of test cases to be run in a way that faults are detected as soon as possible. This enables faster feedback about the system under test and lets developers begin correcting faults earlier. Hence, it could provide faster evidence that quality objectives were not met and the assurance that those tests with greatest fault detection ability will have been executed if testing is halted [26].

Figure 2 depicts a rough overview of the general SPL testing process and how our prioritization approach fits on it. First, the variability model (usually a feature model) is inspected and the set of products to be tested is selected. The selection could be done either manually (e.g. selecting the product portfolio of the company) or automatically (e.g. using t-wise). Once the suite is selected, specific test cases should be designed for each product under test. Then, the set of products to be tested could be prioritized according to multiple criteria determining the execution order of the test cases. Some of the criteria may need analyzing the feature model or using feedback from previous test executions during regression testing. A point to remark is that prioritization does not require creating new test cases, just reordering the existing ones. As a result, prioritization could be re-executed as many times as needed with different criteria. For instance, during the initial stages of development products could be reordered to maximize feature coverage and during regression testing they could be reordered to detect critical faults as soon as possible, e.g. those causing failures in a higher number of products. The prioritization criteria proposed are presented in the next sections.

\section{A. CTCR prioritization criterion}

This criterion is based on the Cross-Tree-Constraints Ratio (CTCR) defined in Section II-A. The CTCR metric has been used to calculate the complexity of feature models and it is correlated with the possibility and ease of change in a model when modifications are necessary [3]. This metric inspired us to define the CTCR prioritization criterion as a way to identify the more complex products in terms of the degree of involvement in the constraints of their features. We hypothesize that this criterion can reduce testing effort while retaining a good fault detection rate by testing earlier the more complex products in terms of constraints.

Given a product $p$ and a feature model $f m$, we define the
CTCR criterion as follows:

$$
\operatorname{CTCR}(p, f m)=\frac{\# \text { constraintsfeatures }(p, f m)}{\# \text { features }(p)}
$$

\#constraintsfeatures $(p, \mathrm{fm})$ denotes the number of distinct features in $p$ involved in constraints and \# features $(p)$ is the number of total features in product $p$. This formula returns a value that indicates the complexity of product $p$ in terms of features involved in constraints.

As an example, the CTCR prioritization value of the products P4 and P6 presented in Section II-A is calculated as follows:

$$
\begin{gathered}
C T C R(P 4, f m)=2 / 7=0.29 \\
C T C R(P 6, f m)=0
\end{gathered}
$$

In P4, the Credit Card and High Security features share an include constraint and also High Security feature has an exclude constraint with Public report. However, the features in P6 do not involve any constraints. Thus, product P4 will be tested earlier than product $\mathrm{P} 6$ according to the $\mathrm{CTCR}$ values i.e. $C T C R(P 4, f m)>C T C R(P 6, f m)$.

\section{B. CoC prioritization criterion}

The Coefficient of Connectivity-Density (CoC) metric, presented in Section II-A, was proposed to calculate the complexity of a feature model in terms of the number of edges and constraints of the model [3]. We propose to adapt this metric for SPL products and use it as a test case prioritization criterion. The goal is to measure the complexity of products according to their $\mathrm{CoC}$ and give higher priority to those ones with higher complexity.

Given a product $p$ and a feature model $\mathrm{fm}$, we define the $\mathrm{CoC}$ of a product as shown below:

$$
C o C(p, f m)=\frac{\# \text { edges }(p, f m)}{\# \text { features }(p)}
$$

\#edges $(\mathrm{p}, \mathrm{fm})$ denotes the number of edges (parent-child connections plus cross-tree constraints) among the features in product $p$. This formula returns a value that indicates the complexity of $p$ based on the $\mathrm{CoC}$ metric.

As an example, the $C o C$ value of the products $\mathrm{P} 7$ and $\mathrm{P} 9$ presented in Section II-A is calculated as follows:

$$
\begin{gathered}
C o C(P 7, f m)=8 / 8=1 \\
C o C(P 9, f m)=9 / 8=1.13
\end{gathered}
$$

In P7, the E-Shop feature is connected with edges to four features (Catalogue, Payment, Security and Search). Also, Payment is connected to the Bank Transfer feature, Security to the Standard Security feature, Search to Public report and Public report has an exclude constraint with High Security. Note that the exclude constraint is considered because it is being fulfilled by this product since it includes Public report feature and not High Security feature. Thus, P9 has higher priority than P7 and therefore it would be tested first. 


\section{VC\&CC prioritization criterion}

In [10], the authors presented a genetic algorithm for the generation of SPL products with an acceptable tradeoff between fault coverage and feature coverage. As part of their algorithm, they proposed a fitness function to measure the ability of a product to exercise features and reveal faults, i.e. the higher the value of the function, the better the product. This function is presented below:

$$
V C \& C C(p, f m)=\sqrt{v c(p, f m)^{2}+c c(p, f m)^{2}}
$$

$v c(p, f m)$ calculates the variability coverage of a product $p$ of the model $f m$ and $c c(p, f m)$ represents the cyclomatic complexity of $p$ (c.f. Section II-A).

Since this function has been successfully applied to SPL test case selection, we propose to explore its applicability for test case prioritization. According to this criterion, those products with higher values for the function are assumed to be more effective in revealing faults and will be tested first.

As an example, the VC\&CC value of the products P3 and P6 presented in Section II-A is calculated as follows:

$$
\begin{aligned}
& V C \& C C(P 3, f m)=\sqrt{3^{2}+2^{2}}=\sqrt{9+4}=3.6 \\
& V C \& C C(P 6, f m)=\sqrt{4^{2}+0^{2}}=\sqrt{16+0}=4
\end{aligned}
$$

In product P3, E-Shop, Payment and Security features are variation points. Also, P3 presents a require constraint with Credit Card and High Security features and an exclude constraint between High Security and Public report. According to this criterion, product P6 would be tested earlier than product $\mathrm{P} 3, V C \& C C(P 6)>V C \& C C(P 3)$.

\section{Commonality prioritization criterion}

We define a commonality-based prioritization criterion that calculates the degree of reusability of products features. That is, the features that have higher commonality and the products that contain them will be given priority to be tested. This enables the early detection of faults in highly reused features that affect to a high portion of the products providing faster feedback and letting software engineers begin correcting critical faults earlier.

Given a product $p$ and a feature model $f m$, we define the Commonality criterion as follow.

$$
\operatorname{Comm}(p, f m)=\sum_{i=1}^{\# \text { features }(p)}(\operatorname{Comm}(\text { fi })) / \# \text { features }(p)
$$

$f i$ denotes a feature of product $p$. The range of this measure is $[0,1]$. Roughly speaking, the priority of a product is calculated by summing up the commonality of its features. The sum is then normalized according to the number of product features.

As an example, the Commonality values of the products $\mathrm{P} 1$ and P2 presented in Section II-A are calculated as follows:

$$
\begin{gathered}
\operatorname{Comm}(\text { P } 1, \mathrm{fm})=(\operatorname{Comm}(\text { EShop })+\operatorname{Comm}(\text { Catalogue }) \\
+\operatorname{Comm}(\text { Payment })+\operatorname{Comm}(\text { Bank Transfer })+\operatorname{Comm}(\text { High }) \\
+\operatorname{Comm}(\text { Security })) / 6=((9+9+9+7+9+6) / 9) / 6=0.91
\end{gathered}
$$

$$
\operatorname{Comm}(P 2, f m)=((9+9+9+7+9+3) / 9) / 6=0.85
$$

Based on these results, P1 would appear before than P2 in the prioritized list of products and would be tested first.

\section{E. Dissimilarity prioritization criterion}

A (dis)similarity measure is used for comparing similarity (diversity) between a pair of test cases. Hemmati et al. [11] and Henard et al. [12] investigated ways to select an affordable subset with maximum fault detection rate by maximizing diversity among test cases using the dissimilarity measure. The results obtained in those papers suggested that two dissimilar test cases have a higher fault detection rate than similar ones since the former ones are more likely to cover more components than the latter.

In this context, we propose to prioritize the test cases based on this dissimilarity metric, testing the most different products first, assuring a higher feature coverage and a higher fault detection rate. In order to measure the diversity between two products, we use the Jaccard distance that compare similarity of sample sets [29]. Jaccard distance is defined as the size of the intersection divided by the size of the union of the sample sets. In our context, each set represents a product containing a set of features. Thus, we choose first the two more dissimilar products (i.e. the products with the highest distance between them) and we add them to a list. Then, we continue adding the products with the highest distance between them until all products have been added to the list. The resulting list of products represents the order of products to be tested.

Given two products $p_{a}$ and $p_{b}$, we define the Dissimilarity formula as follows:

$$
\operatorname{Dissimilarity}\left(p_{a}, p_{b}\right)=1-\frac{\left|p_{a} \bigcap p_{b}\right|}{\left|p_{a} \bigcup p_{b}\right|}
$$

$p_{a}$ and $p_{b}$ represent different set of features (i.e. products). The resulting distance varies between 0 and 1 , where 0 denotes that the products $p_{a}$ and $p_{b}$ are the same and 1 indicates that $p_{a}$ and $p_{b}$ share no features.

The dissimilarity values of the products P1, P7 and P8 presented in Section II-A are calculated as follows:

$$
\begin{aligned}
& \operatorname{Dissimilarity}(P 1, P 7)=1-5 / 9=0.44 \\
& \text { Dissimilarity }(P 7, P 8)=1-5 / 10=0.5
\end{aligned}
$$

For example, regarding to the distance between P1 and P7, they have 5 features in common (E-Shop, Catalogue, Payment, Bank Transfer and Security) out of 9 total features (the previous five plus High, Standard, Search, Public report). P7 and P8 present greater distance between them than P7 and P1. Thus, P7 and P8 would be tested earlier than P1.

\section{EVALUATION}

In this section, we present two experiments to answer the following research questions:

RQ1: Is the order in which SPL products are tested relevant? RQ2: Are the prioritization criteria presented in Section 2 effective at improving the rate of early fault detection of SPL test suites? 
RQ3: Can our prioritization approach improve the rate of early fault detection of current test selection techniques based on combinatorial testing?

We begin by describing our experimental settings and then we explain the experimental results.

\section{A. Experimental settings}

In order to assess our approach, we developed a prototype implementation for each prioritization criterion. Our prototype takes an SPL test suite and a feature model as inputs and generates an ordered set of test cases according to the prioritization criterion selected. We used the SPLAR tool [18] for the analysis of feature models. All the performed experiments were implemented using Java 1.6. We ran our tests on a Linux CentOS release 6.3 machine equipped with an Intel Xeon X5560@2.8Ghz microprocessor and 4 GB of RAM memory.

1) Models: For our experiments we randomly selected 7 feature models of various sizes from the SPLOT repository [19]. Also, we generated 8 random models with up to 500 features using the BeTTy online feature model generator [27]. Table II lists the characteristics of the models. For each model, the name, the number of features and products and the CTCR are presented.

\begin{tabular}{lcccc}
\hline Name & Features & Products & CTCR & Faults \\
\hline Web portal & 43 & 2120800 & $25 \%$ & 4 \\
\hline Video player & 71 & $4,5 \cdot 10^{13}$ & $0 \%$ & 4 \\
\hline Car selection & 72 & $3 \cdot 10^{8}$ & $31 \%$ & 4 \\
\hline Model transf. & 88 & $1 \cdot 10^{12}$ & $0 \%$ & 8 \\
\hline Fm test & 168 & $1,9 \cdot 10^{24}$ & $28 \%$ & 16 \\
\hline Printers & 172 & $1,14 \cdot 10^{27}$ & $0 \%$ & 16 \\
\hline Electronic shop & 290 & $4,52 \cdot 10^{49}$ & $11 \%$ & 28 \\
\hline Random1 & 300 & $7,65 \cdot 10^{39}$ & $8 \%$ & 28 \\
\hline Random2 & 300 & $1,65 \cdot 10^{32}$ & $5 \%$ & 28 \\
\hline Random3 & 350 & $7,41 \cdot 10^{37}$ & $10 \%$ & 32 \\
\hline Random4 & 400 & $3,06 \cdot 10^{44}$ & $10 \%$ & 40 \\
\hline Random5 & 450 & $3,80 \cdot 10^{54}$ & $0 \%$ & 44 \\
\hline Random6 & 450 & $1,03 \cdot 10^{48}$ & $5 \%$ & 44 \\
\hline Random7 & 500 & $4,97 \cdot 10^{36}$ & $5 \%$ & 48 \\
\hline Random8 & 500 & $2,21 \cdot 10^{58}$ & $5 \%$ & 48 \\
\hline
\end{tabular}

TABLE II: Feature models used in our experiments

2) Fault generator: To measure the effectiveness of our proposal, we evaluated the ability of our test case prioritization criteria to detect faults in the SPL under test. For this purpose, we implemented a fault generator for feature models. This generator is based on the fault simulator presented by Bagueri et al. which has been used in several works to evaluate the fault detection rate of SPL test suites [2], [10]. Our fault generator simulates faults in n-tuples of features with $n \in[1,4]$ (where $n$ is a natural integer giving the number of features present in the n-tuple). Faults in n-tuples simulate interaction faults which require the presence of a set of features to be revealed. Studies show that faults caused by the interaction of between
2 and 4 features are frequent in practice [6]. The number of faults seeded on each model is equal to $m / 10$ being $m$ the number of model features. This information is detailed in the last column of Table II. Each type of fault was introduced in the same proportion, i.e. $25 \%$ in single features, $25 \%$ in 2-tuples of features, $25 \%$ in 3-tuples of features and $25 \%$ in 4-tuples of features. Hence, our generator receives a feature model as input and returns a random list of faulty feature sets as output. For instance, for the model in Figure 1 the faults seeded could be given as follows: $\{\{$ High $\}\{$ Credit Card,Search $\}\}$ representing a fault in the feature High Security and another fault caused by the interaction between the Credit Card and Search features.

3) Evaluation metric: In order to evaluate how quickly faults are detected during testing we used the Average Percentage of Faults Detected (APFD) metric [23], [25], [26], [28]. The APFD metric measures the weighted average of the percentage of faults detected during the execution of the test suite. To formally illustrate APFD, let $T$ be a test suite which contains $n$ test cases, and let $F$ be a set of $m$ faults revealed by $T$. Let $T F i$ be the position of the first test case in ordering $T$ ' of $T$ which reveals the fault $i$. According to [8], the APFD metric for the test suite $T$ ' could be given by the equation:

$$
A P F D=1-\frac{T F_{1}+T F_{2}+\ldots+T F_{n}}{n \times m}+\frac{1}{2 n}
$$

APFD value ranges from 0 to 1 . A prioritized test suite with higher APFD value has faster fault detection rates than those with lower APFD values. For example, consider a test suite of 4 test cases, T1 through T4, and 5 faults detected by those test cases, as shown in Table III. Consider two orderings of these test cases, ordering O1: T1,T2,T3,T4 and ordering $\mathrm{O} 2$ : $\mathrm{T} 3, \mathrm{~T} 2, \mathrm{~T} 4, \mathrm{~T} 1$. According to the previous APFD equation, ordering O1 produces an APFD of $58 \%$ $\left(1-\frac{1+1+2+3+4}{4 \times 5}+\frac{1}{2 \times 4}=0.58\right)$ and ordering O2 an APFD of $78 \%\left(1-\frac{1+1+1+1+3}{4 \times 5}+\frac{1}{2 \times 4}=0.78\right)$, being $\mathrm{O} 2$ much faster detecting faults than $\mathrm{O} 1$.

\begin{tabular}{lccccc}
\hline Tests/Faults & F1 & F2 & F3 & F4 & F5 \\
\hline $\mathrm{T} 1$ & $\mathrm{X}$ & $\mathrm{X}$ & & & \\
\hline $\mathrm{T} 2$ & $\mathrm{X}$ & & $\mathrm{X}$ & & \\
\hline $\mathrm{T} 3$ & $\mathrm{X}$ & $\mathrm{X}$ & $\mathrm{X}$ & $\mathrm{X}$ & \\
\hline $\mathrm{T} 4$ & & & & & $\mathrm{X}$ \\
\hline
\end{tabular}

TABLE III: Test suite and faults exposed

\section{B. Experiment 1. Prioritizing SPL test suites}

In order to answer $R Q 1$ and $R Q 2$, we checked the impact on the rate of early fault detection of the prioritization criteria defined in Section III. The experimental setup and the results are next reported.

Experimental setup. For each model presented in Table II, we performed several steps. First, we used our fault generator to simulate faults in the SPL obtaining as a result a list of faulty features sets. Then, we randomly generated a test suite using SPLAR. The suite was composed of between 100 and 500 products depending on the size of the model. This step simulates the manual tests selection of an SPL engineer who could choose the products to be tested following multiple criteria: cost, release plan, users requests, marketing strategy, 
etc. For each fault in the model, we made sure that there was at least one product in the suite detecting it, i.e. the suite detected $100 \%$ of the faults. Once generated, the suite was ordered according to the prioritization criteria defined in Section III resulting in six total test suites, one random suite and five prioritized suites. Finally, we measured how fast the faults were detected by each suite calculating their APFD values. For the random suite the APFD was calculated as the average of 10 random orderings to avoid the effects of chance.

Experimental results. Table IV depicts the size of the test suites and the APFD values obtained by the random and the five prioritized suites for each model. The best value on each row is highlighted in boldface. Also, mean values are shown in the final row. As illustrated, there are significant differences on the APFD average values ranging from the $74.9 \%$ of the Commonality-ordered suite to the $96.5 \%$ reached by the VC\&CC-ordered suite. These differences are even more noticeable in individual cases. For the model "Random3", for instance, the difference between the random and VC\&CCordered suite is 33.3 points, i.e. from $63.9 \%$ to $97.2 \%$. The best average results were obtained by the VC\&CC criterion with $96.5 \%$, followed by CoC $(90.6 \%)$, Dissimilarity $(87.4 \%)$, CTC $(84.5 \%)$, random criterion $(77.4 \%)$ and Commonality (74.9\%). Interestingly, the APFD values obtained by the random suites in the models of lower size were remarkably high, e.g. $A P F D($ Videoplayer $)=92.0 \%$. We found that this was due to the low number of faults seeded and to the size of the models that made the faults easily detectable using just a few tests. In the eight largest models, however, the difference between the random suite APFDs (63.9\%-77.5\%) and the ones of the prioritized suites $(91.5 \%-98.2 \%)$ was noticeable. This suggests that our approach is especially helpful in large test spaces. Finally, we may remark that all the proposed prioritization criteria except Commonality improved the mean value obtained by the random ordering. In fact, for all models at least several of the prioritized suites improved the random APFD values.

Figure 3 shows the percentage of detected faults versus the fraction of the test suite used for the model "Random3". Roughly speaking, the graphs show how the APFD value evolves as the test suite is exercised. It is noteworthy that the VC\&CC-ordered suite, for instance, detected all the faults (32) by using just $15 \%$ of the suite, i.e. 75 test cases out of 500 with the highest priority. Another example is the Dissimilarityordered suite that detected all the faults by using only the $45 \%$ of the suite. The random suite, however, required using $95 \%$ of the test cases to detect exactly the same faults. This behaviour was also observed in the rest of the models under study. In real scenarios, with a higher number of faults and time-consuming executions, this acceleration in the detection of faults could imply important saving in terms of debugging efforts.

The results obtained answer positively to $R Q 1$ and $R Q 2$. Regarding $R Q 1$, the results show that the order in which tests are run is definitely relevant and can have a clear impact on the early fault detection rate of an SPL suite. Regarding $R Q 2$, the results suggest that the presented ordering criteria, especially VC\&CC, CoC and Dissimilarity could be effective at improving the rate of early fault detection of SPL test suites.

\begin{tabular}{lccccccc}
\hline \multirow{2}{*}{ FM } & \multirow{2}{*}{ Suite size } & \multicolumn{7}{c}{ APFD } \\
\cline { 3 - 8 } & & Random & CoC & CTC & Comm & VC\&CC & Diss \\
\hline Web p. & 100 & 81.5 & 95.8 & 94.3 & 60.0 & $\mathbf{9 9 . 0}$ & 92.3 \\
\hline Car s. & 100 & 83.6 & 96.5 & 94.8 & 92.5 & $\mathbf{9 7 . 8}$ & 90.5 \\
\hline Video p. & 100 & 92.0 & $\mathbf{9 8 . 8}$ & 93.0 & 76.0 & $\mathbf{9 8 . 8}$ & 97.3 \\
\hline Model t. & 100 & 83.3 & 94.8 & 75.5 & 79.9 & $\mathbf{9 5 . 4}$ & 91.5 \\
\hline Fm test & 300 & 85.2 & 87.3 & 83.4 & 84.3 & $\mathbf{9 4 . 3}$ & 93.6 \\
\hline Printers & 300 & 92.9 & 94.1 & $\mathbf{9 8 . 4}$ & 93.9 & 96.3 & 96.5 \\
\hline E. shop & 300 & 90.2 & 93.4 & 92.1 & 87.6 & 96.3 & $\mathbf{9 7 . 0}$ \\
\hline Random1 & 300 & 64.7 & 92.6 & 84.2 & 60.1 & $\mathbf{9 7 . 9}$ & 89.1 \\
\hline Random2 & 300 & 73.1 & 87.9 & 78.6 & 77.5 & $\mathbf{9 8 . 2}$ & 80.4 \\
\hline Random3 & 500 & 63.9 & 79.6 & 70.7 & 80.7 & $\mathbf{9 7 . 2}$ & 85.2 \\
\hline Random4 & 500 & 69.7 & 93.9 & 92.2 & 53.8 & $\mathbf{9 7 . 8}$ & 65.8 \\
\hline Random5 & 500 & 77.5 & $\mathbf{9 1 . 6}$ & 78.4 & 73.7 & 91.5 & 89.1 \\
\hline Random6 & 500 & 69.5 & 83.5 & 73.6 & 43.8 & $\mathbf{9 5 . 1}$ & 88.8 \\
\hline Random7 & 500 & 66.3 & 90.4 & 81.6 & 72.6 & $\mathbf{9 5 . 7}$ & 87.0 \\
\hline Random8 & 500 & 67.3 & 79.3 & 76.3 & 86.3 & $\mathbf{9 5 . 9}$ & 67.0 \\
\hline Average & & $\mathbf{7 7 . 4}$ & $\mathbf{9 0 . 6}$ & $\mathbf{8 4 . 5}$ & $\mathbf{7 4 . 9}$ & $\mathbf{9 6 . 5}$ & $\mathbf{8 7 . 4}$ \\
\hline
\end{tabular}

TABLE IV: APFD for random and prioritized suites

\section{Experiment 2. Prioritization + combinatorial testing}

In order to answer $R Q 3$, we checked whether our prioritization criteria could be used to increase the rate of early fault detection of test suites based on combinatorial selection. The experimental setup and results are next reported.

Experimental setup. The experimental procedure was similar to the one used in Experiment 1. Feature models were seeded with the same faults used in our previous experiment. Then, for each model, we generated a 2-wise test suite using the SPLCAT tool presented by Johansen et al. [13]. As a result, we obtained a list of products covering all the possible pairs of features on each model. Then, we prioritized the list of products according to our five prioritization criteria and we calculated the APFD of the resulting six suites, 2-wise and five prioritized suites. It is noteworthy that SPLCAT uses an implicit prioritization criterion placing first in the list those products that covers the most uncovered pairs of features. This tends to place those products with more features at the top of the list getting fast feature coverage. This approach therefore is likely to increase the fault detection rate and thus it is considered as an extra prioritization approach in our comparison.

Experimental results. The results of this experiment are presented in Table V. For each model, the size of the pairwise test suite, the number of faults detected out of the total number of seeded faults and the APFD values of each ordering are presented. Note that the generated pairwise suites did not detect all the faults seeded on each model. As illustrated, the APFD average values ranged from $55.0 \%$ to $90.7 \%$. As expected, the APFD average value of the pairwise suite $(85.0 \%)$ was higher than the one of the random suite $(77.4 \%)$ in Experiment 1 . This was due to implicit prioritization criterion used by the SPLCAT tool that places at the top of the list those products containing a higher number of uncovered pairs of features, usually the largest products. As in the previous experiment, the best APFD 


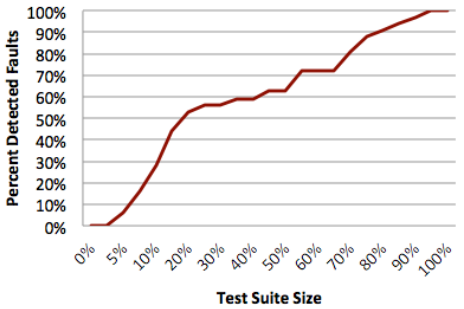

(a) Random APFD

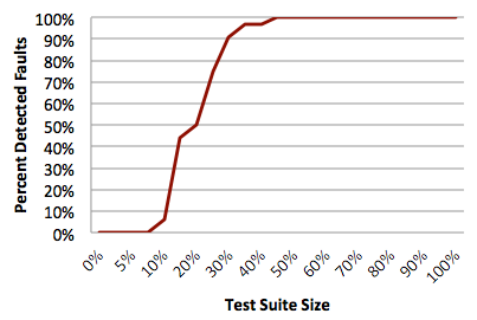

(d) Commonality APFD

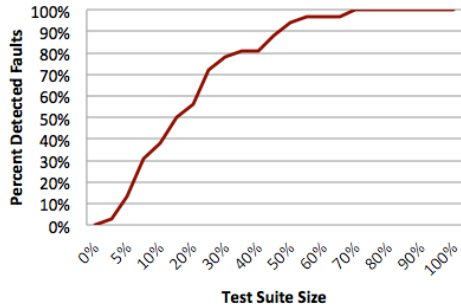

(b) CoC APFD

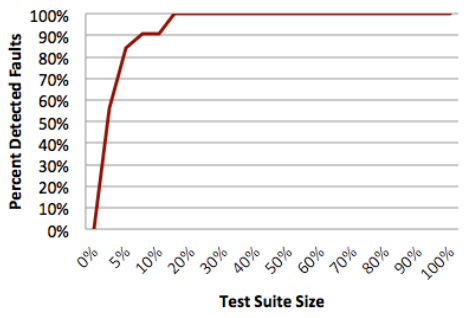

(e) VC\&CC APFD

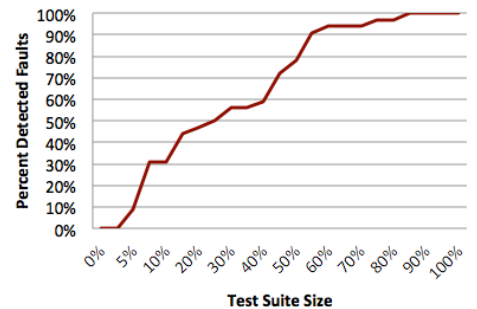

(c) CTC APFD

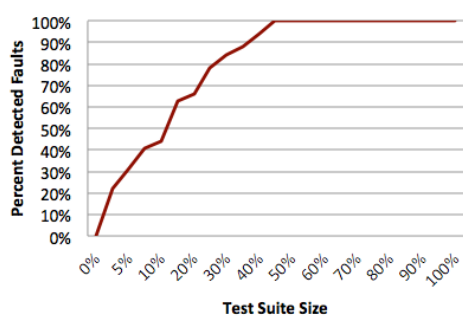

(f) Dissimilarity APFD

Fig. 3: APFD metrics for Random3 model

average results were obtained by the VC\&CC criterion with $90.7 \%$, followed by $\mathrm{CoC}(88.0 \%)$ and Dissimilarity (86.9\%). The pairwise suite got the fourth best APFD average value (85.0\%). The CTC and Commonality prioritization criteria did not get to improve the results of the original suite. In terms of individual values, $\mathrm{CoC}$ got to improve the pairwise APFD values in 13 out of 15 models, VC\&CC in 12 out of 15 models and Dissimilarity in 11 out of 15 . As in our previous experiment, there was not a single model in which the pairwise suite obtained a higher APFD value than all the rest prioritized suites.

Figure 4 shows the percentage of detected faults versus the fraction of suite used for the feature model "Random3". In this example, it is remarkable that VC\&CC-ordered suite detected all the faults with just $20 \%$ of the suite (i.e. 27 tests out of 135). Also, the CoC-ordered and the pairwise suites detected the same faults with only $50 \%$ of the suite. However, the CoCordered suite detected more faults earlier, i.e. the curve of $\mathrm{CoC}$ is slightly steeper than the 2-wise curve. A similar behaviour was observed in the rest of the models under study.

In response to $R Q 3$, our results show that our prioritization criteria can be helpful to increase the rate of early fault detection of the current combinatorial testing techniques.

\section{Threats to VALidity}

In this section we discuss some of the potential threats to the validity of our studies. In order to avoid any bias in the implementation and make our work reproducible, we used a number of validated and publicly available tools. In particular, we used SPLAR [18] for the analysis of feature models, BeTTy [27] for the generation of random feature models, SPLCAT [13] for pairwise test selection and also we implemented a fault generator based on the work of Bagheri et al. [10]. Due to the lack of real SPLs with available test cases, we evaluated our approach by simulating faults in a number of SPLs represented by published and randomly generated models of different sizes.
This may be a threat to our conclusions. However, we may remark that the evaluation of testing approaches using feature models is extensively used in the literature [12], [13], [14]. The use of a fault generator also implies several threats. The type, number and distribution of generated faults could not be the one found in real code. We may emphasize, however, that our generator is based on the fault simulator presented by Bagheri et al. [10] which has been validated in the evaluation of several SPL test case selection approaches [2], [10]. Furthermore, we remark that the characteristics and distribution of faults have a limited impact in our work since we are not interested in how many faults are detected but how fast they are revealed by different orderings of the same test suite.

\section{RELATED WORK}

Concerning the reduction of the number of test cases, Sebastain Oster et al. [20] provided a methodology to apply combinatorial testing to an SPL feature model combining graph transformation and forward checking. In [15], the authors implemented several combinatorial testing techniques adapted to the SPL context. Additionally, Bagheri et al. [2] proposed eight coverage criteria using a grammar-based method to reduce the number of test cases to be tested. Ensan et al. [10] presented a search-based approach using Genetic Algorithms to generate reduced test suites with a high fault-detection capability. As a special case of tests selection, we mention the work performed by Henard et al. [12] that proposed twise covering and prioritization to generate products based on similarity heuristics. In contrast to previous works, we focus on prioritization, not selection. That is, we focus on finding efficient ways of exploring the test space rather than reducing it. To that purpose, we propose a set of prioritization criteria implemented with available tools for the automated analysis of feature models. This makes our approach automated and complementary to all previous works on feature-based test case selection.

Respecting the contributions on test case prioritization, 


\begin{tabular}{lcccccccc}
\hline \multirow{2}{*}{ FM } & \multirow{2}{*}{ Suite size } & \multirow{2}{*}{ Detected faults } & \multicolumn{7}{c}{ APFD } \\
\cline { 3 - 8 } & & & 2wise & CoC & CTC & Comm & VC\&CC & Diss \\
\hline Web p. & 19 & $3 / 4$ & 90.3 & 93.9 & 90.4 & 46.5 & $\mathbf{9 7 . 4}$ & $\mathbf{9 7 . 4}$ \\
\hline Car s. & 24 & $4 / 4$ & 81.2 & $\mathbf{9 0 . 6}$ & $\mathbf{9 0 . 6}$ & 51.0 & 80.2 & 71.9 \\
\hline Video p. & 18 & $4 / 4$ & 76.4 & $\mathbf{9 3 . 1}$ & 76.4 & 22.2 & 83.3 & 83.3 \\
\hline Model t. & 28 & $7 / 8$ & 78.8 & $\mathbf{8 8 . 0}$ & 78.8 & 49.2 & 85.5 & 80.9 \\
\hline FM test & 43 & $15 / 16$ & 85.0 & 68.8 & 55.3 & 53.6 & $\mathbf{9 3 . 9}$ & 75.4 \\
\hline Printers & 129 & $13 / 16$ & 96.2 & $\mathbf{9 7 . 1}$ & 96.2 & 58.0 & 89.5 & 96.8 \\
\hline E shop & 24 & $24 / 28$ & 81.7 & 82.6 & 79.7 & 55.6 & 78.8 & $\mathbf{8 5 . 2}$ \\
\hline Random1 & 124 & $23 / 28$ & 81.1 & 83.4 & 83.6 & 61.7 & $\mathbf{9 6 . 6}$ & 92.2 \\
\hline Random2 & 105 & $25 / 28$ & 86.2 & 91.6 & 90.1 & 52.3 & $\mathbf{9 4 . 8}$ & 90.0 \\
\hline Random3 & 135 & $28 / 32$ & 84.7 & 87.4 & 84.7 & 68.8 & $\mathbf{9 7 . 1}$ & 89.9 \\
\hline Random4 & 178 & $34 / 40$ & 86.9 & 88.8 & 87.8 & 62.9 & $\mathbf{9 5 . 7}$ & 90.7 \\
\hline Random5 & 126 & $38 / 44$ & 86.9 & $\mathbf{9 4 . 9}$ & 86.9 & 61.3 & 94.6 & 80.6 \\
\hline Random6 & 157 & $39 / 44$ & 85.1 & 86.1 & 82.0 & 48.7 & $\mathbf{9 2 . 4}$ & 88.3 \\
\hline Random7 & 253 & $37 / 48$ & 84.9 & 84.2 & 79.2 & 63.1 & 85.8 & $\mathbf{9 1 . 8}$ \\
\hline Random8 & 216 & $40 / 48$ & 89.2 & 90.4 & 86.1 & 70.8 & $\mathbf{9 5 . 1}$ & 88.3 \\
\hline Average & & & $\mathbf{8 5 . 0}$ & $\mathbf{8 8 . 0}$ & $\mathbf{8 3 . 2}$ & $\mathbf{5 5 . 0}$ & $\mathbf{9 0 . 7}$ & $\mathbf{8 6 . 9}$ \\
\hline
\end{tabular}

TABLE V: APDF for 2-wise and prioritized suites

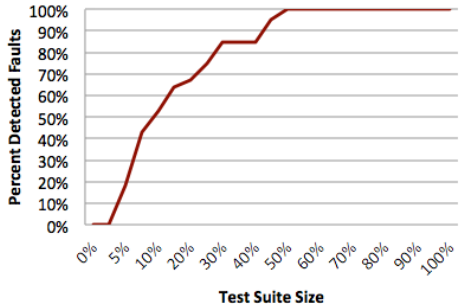

(a) 2-wise APFD

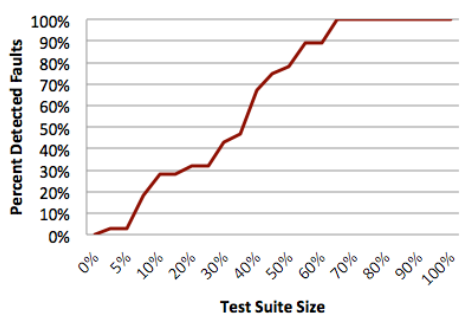

(d) Commonality APFD

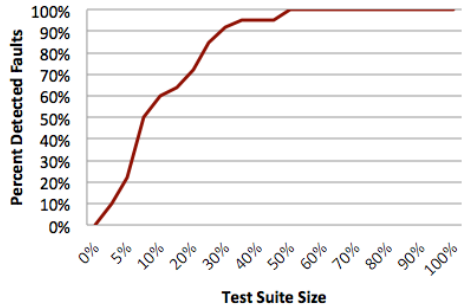

(b) $\mathrm{CoC}$ APFD

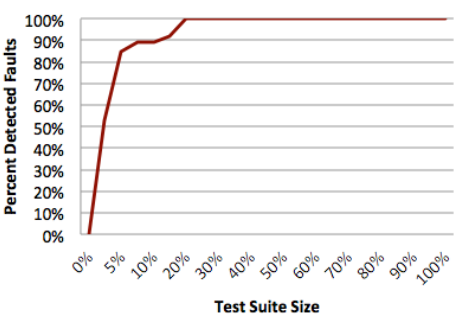

(e) VC\&CC APFD

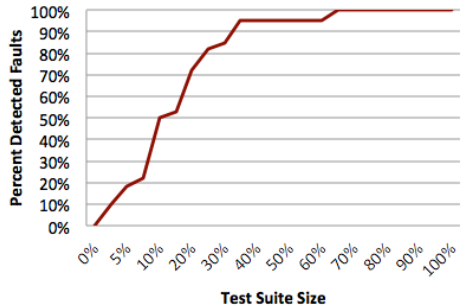

(c) CTC APFD

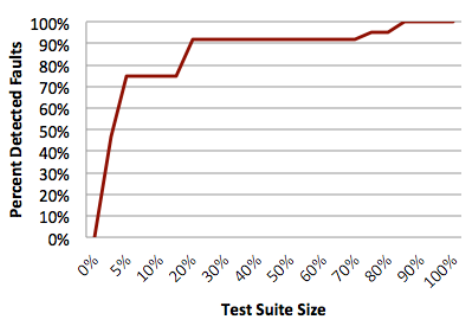

(f) Dissimilarity APFD

Fig. 4: APFD metrics for Random3 model

Rothermel et al. [25] proposed several prioritization techniques for regression testing by using test execution information with the aim of obtain cost-benefits tradeoffs. Zhang et al. [31] used the total and additional prioritization strategies to prioritize based on the total numbers of elements covered per test, and the numbers of additional (not-yet-covered) elements covered per test to increase the rate of fault detection. The work presented in [9] proposed an approach to reduce the SPL test space using a manual goal-oriented method to select and prioritize the most desirable features from feature models. Part of our work is also focused on reflecting the more relevant features of an SPL, however, we propose different prioritization criteria as the complexity of the features, the degree of reusability or the dissimilarity among the products features. These criteria are based on standard metrics for the analysis of feature models and therefore are fully automated.

Another works about prioritization of configurable systems are those presented in [23], [28]. In [23], Qu et al. examined several Combinatorial Iteration Testing prioritization techniques and compared them with a re-generation/prioritization approach (i.e. approach that combined generation and prioritization). Srikanth et al. [28] studied the prioritization driven not only by fault detection but also by the cost of configuration and setup time. In our work, we also present an approach that can combine combinatorial testing and different prioritization criteria to detect faults faster. However, we work within the 
realm of SPLs and in particular we adapt our approach to feature models since they are widely used for variability modelling in SPLs.

\section{CONCLUSIONS AND FUTURE WORK}

In this paper, we presented a test case prioritization approach for SPLs. In particular, we proposed five prioritization criteria to schedule test execution in an order that attempt to accelerate the detection of faults providing faster feedback and reducing debugging efforts. These prioritization criteria are based on standard techniques and metrics for the analysis of feature models and therefore are fully automated. The evaluation results show that there are significant differences in the rate of early fault detection provided by different prioritization criteria. Also, the results show that some of the criteria proposed may contribute to accelerate the detection of faults of both random and pairwise-based SPL test suites. This suggests that our work could be a nice complement for current techniques for test case selection. To the best of our knowledge, our work is the first considering not only which SPL products should be tested but how they should be tested. The main conclusion of this work is that the order in which SPL test cases are run does matter.

Many challenges remain for our future work. First and foremost, we plan to further validate our approach on the source code of real ecosystems such as FaMa and Eclipse. Also, we plan to work on new prioritization criteria exploiting the analysis of non-functional properties, e.g. order tests according to their cost. Test case prioritization techniques have shown to be especially helpful during regression testing. We also intend to work on that direction by defining prioritization criteria based on the feedback from previous tests.

\section{MATERIAL}

Our test case prioritization tool together with the feature models and the seeded faults used in our evaluation are available at www.isa.us.es/ isaweb/anabsanchez/material.zip

\section{ACKNOWLEDGMENTS}

This work was partially supported by the European Commission (FEDER), the Spanish and the Andalusian R\&D\&I programmes (grants TIN2009-07366 (SETI), TIN2012-32273 (TAPAS), TIC-5906 (THEOS)).

\section{REFERENCES}

[1] AHEAD Tool Suite. http://www.cs.utexas.edu/users/schwartz/ATS.html, accessed April 2013.

[2] E. Bagheri, F. Ensan, and D. Gasevic. Grammar-based test generation for software product line feature models. In Conference of the Centre for Advanced Studies on Collaborative Research, 2012.

[3] E. Bagheri and D. Gasevic. Assessing the maintainability of software product line feature models using structural metrics. Software Quality Control, 2011.

[4] D. Benavides, S. Segura, and A. Ruiz-Cortés. Automated analyses of feature models 20 years later: A literature review. Information Systems, 2010

[5] C. Catal and D. Mishra. Test case prioritization: a systematic mapping study. Software Quality Jorunal, 2012.

[6] D. R. W. D. Richard Kuhn and A. M. Gallo. Software fault interactions and implications for software testing. Transactions on Software Engineering, 30, 2004
[7] I. do Carmo Machado, J. D. McGregor, and E. S. de Almeida. Strategies for testing products in software product lines. SIGSOFT Software Engineering, 2012.

[8] S. Elbaum, G. Rothermel, S. Kanduri, and A. G. Malishevsky. Selecting a cost-effective test case prioritization technique. Software Quality Journal, 2004

[9] A. Ensan, E. Bagheri, M. Asadi, D. Gasevic, and Y. Biletskiy. Goaloriented test case selection and prioritization for product line feature models. In Conference Information Technology:New Generations, 2011.

[10] F. Ensan, E. Bagheri, and D. Gasevic. Evolutionary search-based test generation for software product line feature models. In Conference on Advanced Information Systems Engineering (CAiSE'12), 2012.

[11] H. Hemmati and L. Briand. An industrial investigation of similarity measures for model-based test case selection. In ISSRE, 2010

[12] C. Henard, M. Papadakis, G. Perrouin, J. Klein, P. Heymans, and Y. L. Traon. Bypassing the combinatorial explosion: Using similarity to generate and prioritize t-wise test suites for large software product lines. Technical report, 2012.

[13] M. F. Johansen, O. Haugen, and F. Fleurey. Properties of realistic feature models make combinatorial testing of product lines feasible. In MODELS, 2011

[14] M. F. Johansen, O. Haugen, and F. Fleurey. An algorithm for generating t-wise covering arrays from large feature models. In SPLC, 2012.

[15] B. P. Lamancha and M. P. Usaola. Testing product generation in software product lines using pairwise for feature coverage. In International conference on Testing Software and Systems, 2010.

[16] Z. Li, M. Harman, and R. M. Hierons. Search algorithms for regression test case prioritization. IEEE Transactions on Software Engineering, 2007

[17] M. M., W. A., and C. K. Sat-based analysis of feature models is easy. In Proceedings of the Sofware Product Line Conference, 2009.

[18] M. Mendonca. Efficient Reasoning Techniques for Large Scale Feature Models. PhD thesis, University of Waterloo, 2009.

[19] M. Mendonca, M. Branco, and D. Cowan. S.p.l.o.t. - software product lines online tools. In OOPSLA, 2009.

[20] S. Oster, F. Markert, and P. Ritter. Automated incremental pairwise testing of software product lines. In SPLC, 2010.

[21] G. Perrouin, S. Oster, S. Sen, J. Klein, B. Budry, and Y. le Traon. Pairwise testing for software product lines: comparison of two approaches. Software Quality Journal, 2011.

[22] G. Perrouin, S. Sen, J. Klein, B. Baudry, and Y. le Traon. Automated and scalable t-wise test case generation strategies for software product lines. In Conference Software Testing, Verification and Validation, 2010.

[23] X. Qu, M. B. Cohen, and K. M. Woolf. Combinatorial interaction regression testing: A study of test case generation and prioritization. 2007.

[24] G. Rothermel, R. Untch, C. Chu, and M. Harrold. Test case prioritization: An empirical study. In Conference Software Maintenance, 1999.

[25] G. Rothermel, R. Untch, C. Chu, and M. Harrold. Prioritizing test cases for regression testing. IEEE Trans. Software Eng, 27:929-948, 2001.

[26] A. G. M. Sebastian Elbaum and G. Rothermel. Test case prioritization: A family of empirical studies. Transactions on Software Engineering, 2002.

[27] S. Segura, J. Galindo, D. Benavides, J. Parejo, and A. Ruiz-Cortés. Betty: Benchmarking and testing on the automated analysis of feature models. In International Workshop on Variability Modelling of Software-intensive Systems, 2012.

[28] H. Srikanth, M. B. Cohen, and X. Qu. Reducing field failures in system configurable software: Cost-based prioritization. 2009.

[29] P. N. Tan, M. Steinbach, and V. Kumar. Introduction to Data Mining. Addison Wesley, 2006

[30] P. Trinidad, D. Benavides, A. Ruiz-Cortés, S. Segura, and A. Jimenez Fama framework. In Software Product Line Conference, 2008.

[31] L. Zhang, D. Hao, L. Zhang, G. Rothermel, and H. Mei. Bridging the gap between the total and additional test-case prioritization strategies. In ICSE, 2013. 